\title{
Matrix Metalloproteinase Gene Polymorphisms in Periodontitis in Taiwanese Population
}

\author{
Chi-Cheng Tsai ${ }^{1,2 *}$, Yu-Shian $\mathrm{Chou}^{4}$, Ying-Chu Lin ${ }^{3}$, Ya-Ping $\mathrm{Ho}^{3,4}$, Yi-Min $\mathrm{Wu}^{3,4}$ and Kun-Yen $\mathrm{Ho}^{3,4}$ \\ ${ }^{1}$ School of Dentistry, College of Oral Medicine, Chung Shan Medical University, Taiwan \\ ${ }^{2}$ Department of Dentistry, Chung Shan Medical University Hospital, Taiwan \\ ${ }^{3}$ School of Dentistry, College of Dental Medicine, Kaohsiung Medical University, Taiwan \\ ${ }^{4}$ Department of Dentistry, Kaohsiung Medical University Hospital, Taiwan \\ *Corresponding author: Chi-Cheng Tsai, School of Dentistry, College of Oral Medicine, University Hospital, Chung Shan Medical University, Taiwan
}

Submission: 海 November 07, 2017; Published: 㭗 November 29, 2017

\section{Introduction}

Periodontitis is an inflammatory disease associated with bacterial infections. In severe cases is accompanied by the loss of alveolar bone with eventual exfoliation of the teeth [1]. Although bacteria are essential to the induction of the disease, individual susceptibility to periodontitis seems to be of major importance in determining the manifestation and progression of the disease [2]. Bacterial pathogens involved in periodontal diseases exert a part of their destructive effect by triggering and inducing host cells to elevate their secretion of matrix metalloproteinases (MMPs). MMPs comprise a structurally and functionally related family of proteolytic enzymes, which play an essential role in tissue remodelling and repair associated with development and inflammation [3]. Studies have focused on the role of MMPs in the pathogenesis of periodontal diseases [4-6]. Matrix metalloproteinases appear to be intimately associated with tissue destruction since the levels of the active forms of these enzymes obtained from either crevicular fluid or mouth rinse samples correlate with tissue destruction and, therefore, provide a sensitive means of demonstrating disease activity [7]. MMPs can degrade most of the extracellular matrix, basement membrane, and protective serpents as well as in the modification of cytokine action and activation of osteoclasts [8,9]. Studies show that, in the active stage of periodontitis, degradation of gingival tissues is at least partly caused by MMP activity [10]. Functional activities of MMPs are controlled by serum or by tissue inhibitors of MMPs (TIMPs) [11]. The MMPs may have important roles in the susceptibility to periodontitis and/or its severity [1115]. A recent study of gene expressions of MMPs and TIMPs by Kubota et al. [16] found that increases in MMP and increases in the MMP/TIMP ratio suggest imbalanced degradation and synthesis of the extracellular matrix in gingival tissues affected by periodontitis [16]. This imbalance may be the main cause of the tissue destruction associated with periodontitis. The genetic polymorphisms may influence the innate and adaptive immunity, and may thus be deterministic in disease outcome. Genetic polymorphisms may also be protective for disease including periodontal diseases. In view of this important role of MMPs in periodontal diseases, it has been hypothesized that genetic variations affecting the expression or activity of MMPs influence the susceptibility and/or severity of chronic periodontitis and/or aggressive periodontitis. However, there were limited studies in the genetic variants in periodontitis in Taiwanese, so we had conducted the studies during the past few years. In this review article, we reviewed and summarized the literatures on the matrix metalloproteinase genes as putative risk factors for susceptibility to chronic and aggressive periodontitis in Chinese Han population in Taiwan.

\section{The MMP-1 Gene}

The MMP-1 enzyme is an important proteolytic enzyme that can cleave type I, II and III collagens. The MMP-1 gene is located at chromosome position 11q22 and is expressed in various cells, including fibroblasts, macrophages, and endothelial/epithelial cells. de Souza et al. [17] concluded that the MMP-1 SNP genotype in the promoter region is associated with the severity of chronic periodontitis (CP) in Brazilians [17]. However, Astolfi et al. [18] found that MMP-1 SNPs were unassociated with susceptibility to periodontitis in Brazilian nonsmokers. Istagaki et al. [19] reported that MMP-1 and/ or MMP-3 gene promoter SNPs did not affect susceptibility to aggressive periodontitis (AgP) in Japan population. Hollá et al. [20] reported that SNPs (single nucleotide polymorphisims) of the MMP-1 promoter gene did not substantially affect the etiology of CP in Czech population. Another study of Japan population by Suzuki et al. [21] reported that SNPs of MMP-1 are unrelated to AgP. Cao et al. [22] concluded that SNPs of MMP-1 in the -1607bp promoter region may be associated with severe CP and with generalized AgP in Chinese subjects. Recently $(2011,2012)$, Li et al. [23] as well as Loo et al. [24] reported that the MMP-1 -1067 genotype $(2 \mathrm{G} / 2 \mathrm{G} \mathrm{SNP})$ is related to decreased susceptibility to CP $[23,24]$. Our earlier study of Taiwan population showed no 
association between MMP-1-1607 1G/2G genetic polymorphism (rs1799750) and the risk of AgP or CP [25]. However, a study of small sample size (28 AgP, $25 \mathrm{CP}$ and 22 periodontally healthy/H), $\mathrm{Lu}$ found that patients with AgP had higher $1 \mathrm{G}$ allele (AgP: 66.1\%, CP: $40 \%, \mathrm{H}: 34.1 \%$ ) for MMP-1 SNPs suggesting the MMP-1 polymorphism might be associated with AgP (personal communication and co-investigation).

\section{The MMP-2 and TIMP-2 Genes}

The MMP-2 may contribute to tissue destruction in periodontitis [26]. Hollá et al. [27] found that MMP-2 does not significantly affect susceptibility to chronic periodontitis (CP) or severity of CP in European Caucasians. Suzuki et al. [21] indicated that the SNP of MMP-2 is not associated with either aggressive periodontitis (AgP) or CP in Japanese subjects. Gürkan et al. [28] concluded that MMP-2 $(-735 \mathrm{C} / \mathrm{T})$ is unrelated to susceptibility to $\mathrm{CP}$ in Turkish population, later they reported that MMP-2 -735C/T genotypes are unassociated with generalized AgP [29]. de Souza et al. [30] reported that periodontitis is unassociated with TIMP-2 G-418C genotypes in Brazilians [30]. In contrast, a study of Chinese subjects in Chen et al. reported that, although SNPs of MMP-2 were not associated with generalized AgP, the TIMP-2-418C allele was associated with generalized AgP [31]. The authors hypothesized that an increased $\mathrm{C}$ allele frequency may have contributed to the severity of periodontitis in the generalized AgP group. The associations between the distributions of SNPs in the promoter regions of MMP-2 and TIMP-2 were compared in AgP, CP, and control groups in Taiwan population [32]. The MMP-2 -1306C/T, -735C/T, -790T/G and TIMP-2 -418G/C SNPs were genotyped by PCR-RFLP (polymerase chain reaction-restriction fragment length polymorphism) analysis in 69 and 129 patients with AgP and CP respectively, and in 117 healthy controls. Chi-square tests used to investigate whether the genotypes were associated with periodontitis showed that distributions of the C-1306T, C-735T and T-790G variants did not significantly differ between the AgP and control groups. The frequencies of TIMP-2 G-418C gene polymorphisms in non-smokers had a trend of significant difference among the $\mathrm{AgP}, \mathrm{CP}$ and control groups ( $\mathrm{p}=0.07)$. This suggests that TIMP-2 G-418C gene polymorphisms may be associated with periodontitis in the Taiwan population [32].

\section{The MMP-3 Gene}

Astolfi et al. [18] reported that MMP-3 gene SNPs might be associated with the destruction of periodontal tissues in Brazilian non-smokers [18]. In contrast, Suzuki et al. [21] reported that MMP-3 SNPs were not associated with AgP or with CP in Japanese subjects]. However, recent studies performed in 2011 and 2012 reported that MMP-3-1171 genotype 5A/6A polymorphisms might be associated with decreased susceptibility to CP in Chinese subject $[23,24]$. Further studies are needed to verify the association between MMP-3 polymorphisms and periodontitis.

\section{The MMP-8 Gene}

Matrix metalloproteinase (MMP)-8, a protease that degrades numerous extracellular molecules, has been implicated in the pathogenesis of periodontitis. A chair-side test for detecting MMP-8 has been developed [33]. Detecting MMP-8 in gingival crevicular fluid (GCF) can be a very practical method of diagnosing and monitoring periodontal diseases [34,35]. The MMP-8 polymorphisms may affect susceptibility to periodontitis. We have evaluated the association between periodontitis and MMP-8 $-799 \mathrm{C}>\mathrm{T}$ polymorphisms [36]. Genomic DNA was obtained from 361 CP patients, 96 AgP patients, and 106 periodontally healthy controls. Detection of MMP-8-799C $>\mathrm{T}$ polymorphisms by PCRRFLP showed that the genotype frequency significantly differed between the diseased groups and the control group. Multivariate logistic regression analysis with adjustment for age, gender and smoking indicated that the -799T allele was significantly associated with both AgP (adjusted OR=1.99, $\mathrm{p}=0.04$ ) and CP (adjusted $\mathrm{OR}=1.87, \mathrm{p}=0.007$ ). To avoid the confounding effects of smoking on the association between MMP-8 polymorphisms and periodontitis, the analysis was repeated in non-smokers and revealed statistically significant associations. These associations suggest that, in Taiwan non-smokers, the -799T allele of MMP-8 is significantly associated with the risks of both chronic and aggressive periodontitis [36]. However, further studies in other ethnic populations are needed.

\section{The MMP-9 and MMP-12 Genes}

The MMP-9, an important member of the MMP family, has functional SNPs at DNA sequence position -1562. Activities of the1562C/T and T/T genotypes can increase the risk for inflammatory diseases. Suzuki et al. [18] found that MMP-9 SNPs are not associated with AgP in Japanese subjects [21]. Keles et al. [37] stated that the frequencies of CT+TT genotypes of MMP-9 (-1562C/ $\mathrm{T})$ vs. CC genotype are lower in the control group. These results suggest that the MMP-9 promoter gene SNP might be associated with the severity of generalized CP in Turkish populations but not in Brazilian populations [30]. Gürkan et al. [29] in 2007 and 2008 concluded that the T allele of the MMP-9 $(-1562 \mathrm{C} / \mathrm{T})$ gene may decrease the risk of developing AgP and CP. The SNPs of MMP-12 (357 Asn/Ser) are not associated with AgP [28] and that SNPs of MMP-9-1562C/T and MMP-12 357Asn/Ser are not associated with the susceptibility to severe CP [28]. Chen et al. [31] found that CC is the most common genotype of MMP-9-1562C/T SNP whereas the TT homozygote is very rare and did not significantly differ between the AgP group and control group. Recent studies in 2011 and 2012 have found that MMP-9-1562 genotype (2G/2G SNP) is associated with susceptibility to CP in Chinese subjects. In 2015, we analyzed 69 AgP patients, 129 CP patients, and 117 healthy controls. Group comparisons showed that, in subjects who did not drink alcohol, the $\mathrm{C}$ allele carrying genotype of the MMP-9-1562C/T gene had a significant protective effect against AgP after adjustment for age, gender, betel-quid chewing, and smoking [32].

\section{Conclusion}

In conclusion, within the limitations of this study, some of the polymorphisms in the MMPs gene promoter would appear to be determinants of susceptibility to the development of chronic periodontitis or aggressive periodontitis in Taiwanese individuals. Increased expression of MMPs mRNA in periodontal 
tissues from patients with periodontitis observed previously together with other mechanisms, possibly a regulatory network between metalloproteinases and their inhibitors because complex inflammatory and remodeling responses are involved in the initiation and progression of periodontitis. However, more studies with larger sample sizes are needed to clarify the association of these polymorphisms with periodontal diseases of our Taiwanese populations (Table 1).

Table 1: Published information on the studied $\mathrm{mmp}$ genes in Taiwanese.

\begin{tabular}{|c|c|}
\hline Reference & Gene and Polymorphisms \\
\hline Cao et al. [22] & MMP-1 [-1607] bp (association: yes for CP and AgP) \\
\hline Loo et al. [24] & MMP-1 2G[-1607]2G (association: yes for CP \& AgP) \\
\hline Chen et al. [31] & $\begin{array}{c}\text { MMP-2 (association: no for AgP); TIMP-2 G[-418]C } \\
\text { (association: yes for AgP) }\end{array}$ \\
\hline Loo et al. [24] & $\begin{array}{c}\text { MMP-3 [-1171]6A (association: yes, decrease risk for } \\
\text { CP) }\end{array}$ \\
\hline Chou et al. [36] & $\begin{array}{c}\text { MMP-8 C[-799]T (association: yes for T allele, OR: 1.99 } \\
\text { in AgP; OR: 1.87 in CP) }\end{array}$ \\
\hline Loo et al. [24] & MMP-9 2G[-1562]2G (association: yes for CP) \\
\hline $\begin{array}{c}\text { Li et al. [23] } \\
\text { MMP-9 2G[-1562]2G (association: yes for CP) }\end{array}$ \\
$\begin{array}{c}\text { Hsiao et al. } \\
\text { [32] }\end{array}$ & $\begin{array}{c}\text { MMP-2 [C-1306T, C-735T, T-790G] (association: no); } \\
\text { MMP-9 [C-1562T] (non-alcohol drinker: protective } \\
\text { against AgP) }\end{array}$ \\
\hline
\end{tabular}

\section{References}

1. Genco RJ, Löe H (1993) The role of systemic conditions and disorders in periodontal disease. Periodontol 2000 2: 98-116.

2. Landi L, Amar S, Polins AS, Dyke VTE (1997) Host mechanisms in the pathogenesis of periodontal disease. Curr Opin Periodontol 4: 3-10.

3. Nagase H, Woessner JF (1999) Matrix metalloproteinases. J Biol Chem 274(31): 21491-21494.

4. Birkedal-Hansen H (1993) Role of matrix metalloproteinases in human periodontal diseases. J Periodontol 64(5 Suppl): 474-484.

5. Ejeil AL, Igondjo-Tchen S, Ghomrasseni S, Pellat B, Godeau G, et al. (2003) Expression of matrix metalloproteinases (MMPs) and tissue inhibitor of metalloproteinases (TIMPs) in healthy and diseased human gingiva. J Periodontol 74(2): 188-195.

6. Chang YC, Yang SF, Lai CC, Liu JY, Hsieh YS, et al. (2002) Regulation of matrix metalloproteinase production by cytokines, pharmacological agents and periodontal pathogens in human periodontal ligament fibroblast cultures. J Periodontal Res 37(3): 196-203.

7. Sodek J, Overall CM (1992) Matrix metalloproteinases in periodontal tissue remodelling. Matrix Suppl 1: 352-362.

8. Makela M, Salo T, Uitto VJ, Larjava H (1994) Matrix metalloproteinases (MMP-2 and MMP-9) of the oral cavity: Cellular origin and relationship to periodontal status. J Dent Res 73(8): 1397-1406.

9. Ryan ME, Golub LM (2000) Modulation of matrix metalloproteinase activities in periodontitis as a treatment strategy. Periodontol 2000 24: 226-238.
10. Price SJ, Greaves DR, Watkins H (2001) Identification of novel, functional genetic variants in the human matrix metalloproteinase-2 gene. J Biol Chem 276(10): 7549-7558.

11. Birkedal-Hansen $H$ (1993) Role of matrix metalloproteinases in human periodontal diseases. J Periodontol 64(5 Suppl): 474-484.

12. Mäkelä M, Salo T, Uitto VJ, Larjava H (1994) Matrix metalloproteinases (MMP-2 and MMP-9) of the oral cavity: cellular origin and relationship to periodontal status. J Dent Res 73(8): 1397-1406.

13. Lee W, Aitken S, Sodek J, McCulloch CA (1995) Evidence of a direct relationship between neutrophil collagenase activity and periodontal tissue destruction in vivo: Role of active enzyme in human periodontitis. J Periodontal Res 30(1): 23-33.

14. Zee VE, Everts V, Beertsen W (1996) Cytokine induced endogenous procollagenase stored in the extracellular matrix of soft connective tissue results in a burst of collagen breakdown following its activation. J Periodontal Res 31(7): 483-488.

15. Ejeil AL, Igondjo-Tchen S, Ghomrasseni S, Pellat B, Godeau G, et al. (2003) Expression of matrix metalloproteinases (MMPs) and tissue inhibitors of metalloproteinases (TIMPs) in healthy and diseased human gingiva. J Periodontol 74(2): 188-195.

16. Kubota T, Itagaki M, Hoshino C, Nagata M, Morozumi T, et al. (2008) Altered gene expression levels of matrix metalloproteinases and their inhibitors in periodontitis-affected gingival tissue. J Periodontol 79(1): 166-173.

17. de Souza AP, Trevilatto PC, Scarel-Caminaga RM, Brito RB, Line SR, et al. (2003) MMP-1 promoter polymorphism: Association with chronic periodontitis severity in a Brazilian population. J Clin Periodontol 30(2): 154-158.

18. Astolfi CM, Shinohara AL, da Silva RA, Santos MC, Line SR, et al. (2006) Genetic polymorphisms in the MMP-1 and MMP-3 gene may contribute to chronic periodontitis in a Brazilian population. J Clin Periodontol 33(10): 699-703.

19. Istagaki M, Kubota T, Tai H, Shimada Y, Morozumi T (2004) Matrix metalloproteinase- 1 and -3 gene promoter polymorphisms in Japanese patients with periodontitis. J Clin Periodontol 31: 764-769.

20. Hollá LI, Jurajda M, Fassmann A, Dvorakova N, Znojil V, et al. (2004) Genetic variations in the matrix metalloproteinase-1 promoter and risk of susceptibility and/or severity of chronic periodontitis in the Czech population. J Clin Periodontol 31(8): 685-690.

21. Suzuki A, Ji G, Numabe Y, Muramatsu M, Gomi K, et al. (2004) Singlenucleotide polymorphisms associated with aggressive periodontitis and severe chronic periodontitis in Japanese. Biochem Biophys Res Commun 317(3): 887-892.

22. Cao Z, Li C, Zhu G (2006) MMP-1 promoter gene polymorphism and susceptibility to chronicperiodontitis in a Chinese population. Tissue Antigens 68(1): 38-43.

23. Li G, Yue Y, Tian Y, Li JL, Wang M, et al. (2012) Association of matrix metalloproteinase (MMP)-1,3, 9, interleukin (IL)-2, 8 and cyclooxygenase (COX)-2 gene polymorphisms with chronic periodontitis in a Chinese population. Cytokine 60(2): 552-560.

24. Loo WT, Wang M, Jin LJ, Cheung MN, Li GR, et al. (2011) Association of matrix metalloproteinase (MMP-1, MMP-3 and MMP-9) and cyclooxygenase-2 gene polymorphisms and their proteins with chronic periodontitis. Arch Oral Biol 56(10): 1081-1090.

25. Shiao YW (2012) Association of MMP-1 gene polymorphisms and periodontitis in a Taiwan Han population. Master's Thesis, Kaohsiung Medical University, Taiwan.

26. Blavier L, Delaissé JM (1995) Matrix metalloproteinases are obligatory for the migration of preosteoclasts to the developing marrow cavity of primitive long bones. J Cell Sci 108(Pt 12): 3649-3659. 
27. Hollá LI, Fassmann A, Vasku A, Goldbergova M, Beranek M, et al. (2005) Genetic variations in the human gelatinase A (matrix metalloproteinase-2) promoter are not associated with susceptibility to, and severity of, chronic periodontitis. J Periodontol 76(7): 1056-1060.

28. Gürkan A, Emingil G, Saygan BH, Atilla G, Cinarcik S, et al. (2008) Gene polymorphisms of matrix metalloproteinase-2,-9 and -12 in periodontal health and severe chronic periodontitis. Arch Oral Biol 53(4): 337-345.

29. Gürkan A, Emingil G, Saygan BH, Atilla G, Cinarcik S, et al. (2007) Matrix metalloproteinase $-2,-9$, and -12 gene polymorphisms in generalized aggressive periodontitis. Journal Periodontol 78(12): 2338-2347.

30. de Souza AP, Trevilatto PC, Scarel-Caminaga RM, de Brito RB, Barros SP, et al. (2005) Analysis of the MMP-9 (C-1562 T) and TIMP-2 (G-418C) gene promoter polymorphisms in patients with chronic periodontitis. J Clin Periodontol 32(2): 207-211.

31. Chen D, Wang Q, Ma ZW, Chen FM, Chen Y, et al. (2007) MMP-2, MMP-9 and TIMP-2 gene polymorphisms in Chinese patients with generalized aggressive periodontitis. J Clin Periodontol 34(5): 384-389.

32. Hsiao YF, Yang LC, Chou YS, Ho YP, Lin YC, et al. (2016) Tsai CC: Matrix metalloproteinase-2, -9(MMP-2,-9) and tissue inhibitor of MMP-
2(TIMP-2) gene polymorphisms in Taiwanese periodontitis patients. J Dent Sci 11(4): 411-418.

33. Mäntylä P, Stenman M, Kinane DF, Tikanoja S, Luoto H, et al. (2003) Gingival crevicular fluid collagenase-2 (MMP-8) test stick for chairside monitoring of periodontitis. J Periodontal Res 38(4): 436-439.

34. Kinane DF, Darby IB, Said S, Luoto H, Sorsa T, et al. (2003) Changes in gingival crevicular fluid matrix metalloproteinase-8 levels during periodontal treatment and maintenance. J Periodontal Res 38(4): 400404.

35. Sorsa T, Tjäderhane L, Salo T (2004) Matrix metalloproteinases (MMPs) in oral diseases. Oral Dis 10(6): 311-318.

36. Chou YH, Ho YP, Lin YC, Hu KF, Yang YH, et al. (2011) MMP-8 -799 C>T genetic polymorphism is associated with the susceptibility to chronic and aggressive periodontitis in Taiwanese. J Clin Periodontol 38(2): 1078-1084.

37. Keles GC, Gunes S, Sumer AP, Sumer M, Kara N, et al. (2006) Association of matrix metalloproteinase- 9 promoter gene polymorphism with chronic periodontitis. J Periodontol 77(9): 1510-1514. 